\title{
Dynamical Arrest of Electron Transfer in Liquid Crystalline Solvents
}

\author{
Vitaliy Kapko and Dmitry V. Matyushov* \\ Department of Chemistry and Biochemistry and the Center for the Early Events in Photosynthesis, \\ Arizona State University, P.O. Box 871604, Tempe, Arizona 85287-1604
}

Received: March 12, 2006; In Final Form: May 3, 2006

\begin{abstract}
We argue that electron transfer reactions in slowly relaxing solvents proceed in the nonergodic regime, making the reaction activation barrier strongly dependent on the solvent dynamics. For typical dielectric relaxation times of polar nematics, electron transfer reactions in the subnanosecond time scale fall into nonergodic regime in which nuclear solvation energies entering the activation barrier are significantly lower than their thermodynamic values. The transition from isotropic to nematic phase results in weak discontinuities of the solvation energies at the transition point and the appearance of solvation anisotropy weakening with increasing solute size. The theory is applied to analyze experimental kinetic data for the electron transfer kinetics in the isotropic phase of 5CB liquid crystalline solvent. We predict that the energy gap law of electron transfer reactions in slowly relaxing solvents is characterized by regions of fast change of the rate at points where the reaction switches between the ergodic and nonergodic regimes. The dependence of the rate on the donoracceptor separation may also be affected in a way of producing low values for the exponential falloff parameter.
\end{abstract}

\section{Introduction}

Electron transfer (ET) reactions have traditionally been studied in isotropic dipolar and nondipolar molecular solvents. The main consequences of the effect of isotropic solvents on the activation barrier of ET are reasonably well understood., The extension of ET theories into the realm of biological enzymatic and photosynthetic reactions ${ }^{3}$ and molecular electron$\mathrm{ics}^{4}$ may require, however, the basic understanding of ET activation in anisotropic media characterized by a preferential direction of dipolar polarization. ${ }^{5}$ Liquid crystals, in particular nematics, provide a model solvent for studying the effects of polarization anisotropy on electronic transitions in molecules. ${ }^{6}$ A theory of activation barrier in liquid crystals will provide a conceptual framework for studying reactions in ordered media such as surfaces, monolayers, and solvents with local or global ferroelectric order. At the same time, liquid crystalline and, more generally, ordered media may potentially offer some advantages in designing artificial photosynthetic devices ${ }^{7}$ and in carrying out redox protein reactions in controlled environments. ${ }^{8}$

In addition to anisotropic polarization, two other properties of liquid crystals are of great interest for studies of ET in complex environments: the appearance of the orientational phase transition and sluggish dielectric relaxation, particularly in the nematic phase. The latter property is useful for studying the effect of slow solvent dynamics on ET, which, for reactions in molecular polar solvents, is normally achieved only by supercooling. Three subjects in application to ET reactions will therefore be the focus of this paper: (i) polarization anisotropy, (ii) orientational phase transition, and (iii) slow dielectric relaxation.

There are very few examples of experimental characterization of ET kinetics in nematic and isotropic phases of liquid crystalline solvents. ${ }^{9-14}$ The generally observed trend for chargeseparation reactions is significantly slower kinetics in liquid crystals, either below or above the IN transition, relative to

* Corresponding author. E-mail: dmitrym@asu.edu. isotropic solvents of comparable polarity.9,11,14 The temperature dependence of ET rates in the isotropic phase above the IN transition temperature is peculiar. Depending on the direction of ET (forward vs backward) and dielectric anisotropy of the liquid crystal, Arrhenius or non-Arrhenius dependence of the rate was observed. ${ }^{10,11}$ This effect, as well as slower kinetics compared to dipolar isotropic solvents, was explained by solvent dynamics controlling the rate of ET. In this regime, the rate is inversely proportional to the dielectric longitudinal relaxation time $\tau_{\mathrm{L}}:^{15,16}$

$$
k_{\mathrm{ET}} \propto \tau_{\mathrm{L}}^{-1}
$$

This interpretation of the observations has several drawbacks. First, it is well established that the competition of the dielectric solvent dynamics with the intramolecular solute dynamics and ballistic solvent relaxation leaves a very narrow window in terms of relaxation time magnitudes for the dynamic solvent effect to be observed. ${ }^{17-19}$ Second, the observation of the non-Arrhenius temperature dependence of the rate is inconsistent with the dielectric data pointing to the Arrhenius temperature dependence of $\tau_{\mathrm{L}}$ both in the isotropic and nematic phases of nematogens..$^{20,21}$

The description of electronic transitions in liquid crystals is complicated by the existence of aligned pseudonematic domains in the isotropic phase several degrees above the isotropicnematic (IN) phase transition. ${ }^{21,22}$ The appearance of such mesoscopic heterogeneities is commonly related to the correlation length $\xi_{\mathrm{Q}}$ of the fluctuations of the nematic order parameter Q. In mean-field theories, the correlation length scales as $\xi_{\mathrm{Q}} \propto$ $\left(T-T_{0}\right)^{-1 / 2}$ on approaching the stability limit of the isotropic phase $T_{0}$ from above. Correspondingly, the relaxation time of the order parameter diverges as

$$
\tau_{\mathrm{Q}} \propto\left(T-T_{0}\right)^{-1}
$$




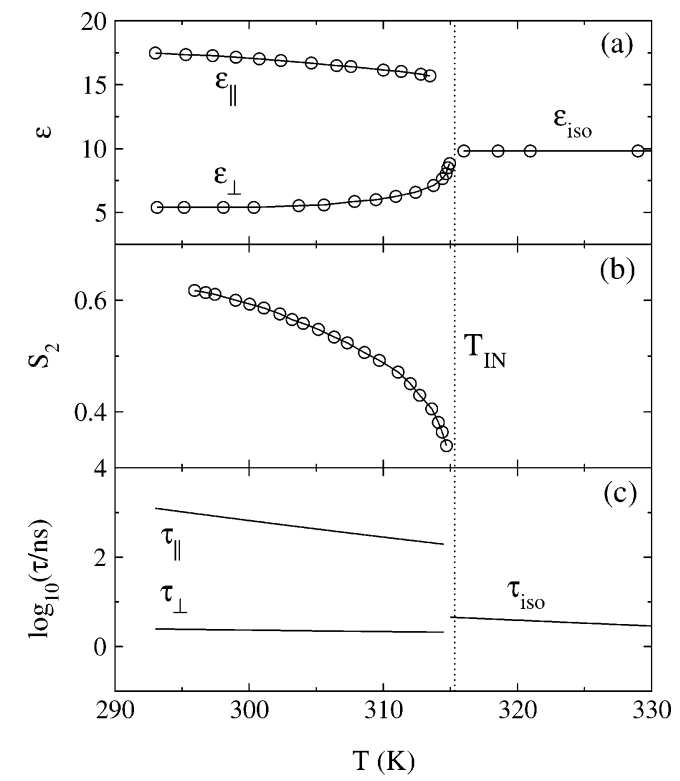

Figure 1. Static dielectric constant ${ }^{20}$ (a), nematic order parameter ${ }^{29}$ (b), and the dielectric relaxation time ${ }^{20}$ (c) vs $T$ for $7 \mathrm{CB}$ nematogen. The dielectric constant splits into the component parallel to the nematic director (II) and the component perpendicular the nematic director $(\perp)$ below the IN transition temperature $T_{\mathrm{IN}}=314.7 \mathrm{~K}^{25}$ (vertical dotted line). The order parameter $S_{2}$ is the largest eigenvalue of the secondrank order parameter $\mathbf{Q}$. The Debye relaxation time in (c) splits into parallel and perpendicular components below $T_{\mathrm{IN}}$. The solid lines in (a) and (b) connect the experimental points and represent fits of the experimental data in (c): ${ }^{20} \tau_{\mathrm{D}}(T)=\tau_{0} \exp (\Delta H / R T), \tau_{0}^{\text {iso }}=2.2 \times 10^{-4}$ $\mathrm{ns}, \tau_{0}^{\|}=2.1 \times 10^{-9} \mathrm{~ns}, \tau_{0}^{\perp}=0.21 \mathrm{~ns}, \Delta H^{\text {iso }}=26.0 \mathrm{~kJ} / \mathrm{mol}, \Delta H^{\|}=$ $66.0 \mathrm{~kJ} / \mathrm{mol}, \Delta H^{\perp}=6.0 \mathrm{~kJ} / \mathrm{mol}$.

resulting in the critical slowing down of the order parameter fluctuations. Transient grating optical Kerr studies ${ }^{23}$ and dynamic light scattering 22 indeed reveal a slow relaxation time in the pretransitional region that follows the mean-field law, along with a temperature-independent relaxation associated with intradomain dynamics.

When combining eqs 1 and 2, it is tempting to assume that the divergent time scale of the nematic order fluctuations is projected into a non-Arrhenius temperature dependence of the rate preexponent. This interpretation, however, contradicts what is known about nematics. Dynamic light scattering or timeresolved Kerr effect techniques provide the correlation function of molecular anisotropic polarizability $\langle\boldsymbol{\alpha}(t) \boldsymbol{\alpha}(0)\rangle .{ }^{24}$ Because this correlation function is a fourth-rank tensor, these measurements are sensitive to the equilibrium average and dynamics of the nematic order parameter $\mathbf{Q}$, which is a second-rank tensor. Optical transitions and ET reactions in dipolar solvents are predominantly affected by the solvent through the coupling of the solute electric field to the solvent dipolar polarization, which is a first-rank tensor. Therefore, pretransitional critical behavior of the nematic order parameter only indirectly affects electronic transitions in molecules dissolved in polar nematics. (Note that spectroscopy and ET in quadrupolar solvents (zero dipole moment, nonzero quadrupole moment) is coupled directly to the nematic order parameter.) Indeed, the vast majority of the dielectric studies suggest Arrhenius temperature dependence of the dielectric relaxation time and Debye spectrum of dielectric loss both above and below the IN transition temperature $T_{\mathrm{IN}}$ (see Figure 1). ${ }^{21,25,26}$ Deviations from the Arrhenius law, resulting in Vogel-Fulcher or power-law temperature dependence of the dielectric relaxation time, are very weak and can be observed only in the derivative of the linear dielectric response $\mathrm{e}^{27,28}$ or from a nonlinear dielectric response. ${ }^{27}$
Given the Arrhenius dielectric relaxation of nematogens, the issue of non-Arrhenius reaction rates seen in experiment ${ }^{10,11}$ remains open. Several types of solvent effects on ET may be considered for reactions in polar nematics. The dielectric constant is anisotropic below the IN transition temperature ${ }^{20,29}$ (Figure 1a). One then may expect anisotropy of all solvation Gibbs energies, entering the rate activation barrier, in respect to the orientation of the donor-acceptor complex. Such an anisotropy indeed follows from the calculations and simulations in the nematic phase of nematogens. ${ }^{30,31}$ However, anisotropy of solvation thermodynamics cannot be explained solely by anisotropy of the dielectric constant because the large size of the molecules of the liquid crystal makes it necessary to include microscopic solvation effects related to a finite length of correlation between the molecular dipoles of the solvent (see below).

The dynamical facet of the problem, in addition to equilibrium solvation thermodynamics, needs also be considered. Dielectric relaxation splits into two components below the IN transition: low-frequency relaxation associated with rotations of molecules around their short axes and high-frequency relaxation related to rotations around long molecular axes (Figure 1c). ${ }^{21}$ The highfrequency relaxation time $\tau_{\perp}$ ( $\perp$ refers to the dielectric response perpendicular to the nematic director) drops at $T_{\mathrm{IN}}$, indicating that rotations around the long axis are less hindered in the nematic phase than in the isotropic phase. On the contrary, the low-frequency relaxation time $\tau_{\|}$(II refers to the response parallel to the nematic director) increases at $T_{\mathrm{IN}}$ by the retardation factor $g_{\|} \simeq 10$ due to the nematic potential hindering rotations away from the nematic director. ${ }^{26,32}$ Even stronger retardation is achieved for transitions to a translationally ordered liquid crystalline phase when the retardation factor may reach a much higher value $\left(g_{\|} \simeq 5000\right.$ for $\mathrm{I}-\mathrm{Sm} \mathrm{B}$ transition $\left.^{33}\right)$. Nematic potential and the large size of molecules making the liquid crystal result in the dielectric relaxation of nematics normally significantly slower than dielectric relaxation of polar isotropic solvents. For instance, the Debye relaxation time in 4,4- $n$-heptylcyanobiphenyl (7CB) nematogen ${ }^{20,21}$ is $\tau_{\mathrm{D}} \simeq 10^{-8}$ s compared to the picosecond time scale of polar isotropic solvents.

Slow relaxation requires new approaches for the effect of solvent dynamics on ET reactions. ${ }^{34-39}$ The Kramers-type description of the solvent effect on chemical reaction rates, which leads to eq 1 , corrects the transition-state theory by incorporating the depletion of the activated-state population by the solvent friction. ${ }^{40}$ This description still assumes that the reaction time, i.e., the time required for the system to climb the activation barrier from the equilibrium bottom of the potential surface, is much longer than the relaxation time of the solvent. The slow relaxation dynamics of nematics put under question the very applicability of the statistical mechanics calculations of the activation barrier, ${ }^{34}$ which is still assumed in the Kramers picture. When the relaxation time of the solvent crosses the observation time of the experiment, which for ET reaction is set by $k_{\mathrm{ET}}{ }^{-1}$ (subnanoseconds for reactions studied in refs 10$12,14)$, the system does not completely explore its phase space and equilibrium solvation is not established. ${ }^{41}$ Under such conditions, the system ergodicity breaks down, and the Gibbs energy of nuclear solvation cannot reach its full equilibrium value on the time-scale of the reaction. ${ }^{34,35,41-45}$ This dynamical arrest of nuclear solvation makes the activation barrier, in contrast to the rate preexponent in eq 1 , dependent on the solvent dynamics. Therefore, in this range of time scales, solvent dynamics still play a significant role in activating electronic transitions, although not as explained by theories of solvent 


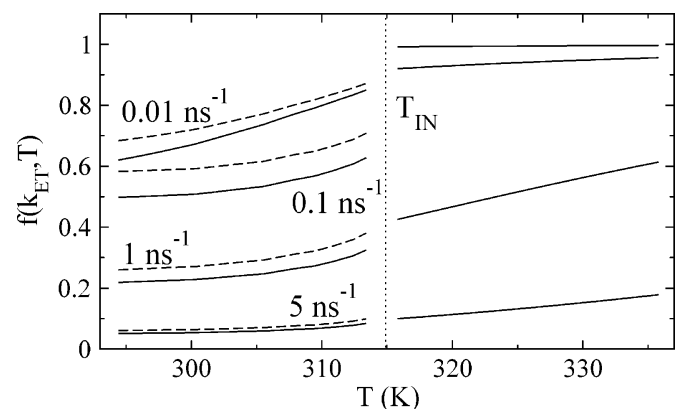

Figure 2. Nonergodicity function $f\left(k_{\mathrm{ET}}, T\right)$ calculated from eqs 3,10 , and 18 with the dielectric relaxation time of 7CB nematogen (Figure $1 c)$ at $k_{\mathrm{ET}}$ values shown on the plot. The solid lines refer to the angle $\theta_{0}=0^{\circ}$ between the direction of the solute dipole moment and the director; the dashed lines refer to $\theta_{0}=90^{\circ}$. The dotted vertical line indicates the IN transition temperature.

dynamics control developed for relatively fast relaxing solvents ${ }^{15,17-19}$ and questioned by more recent studies. ${ }^{17-19}$

To introduce nonergodic nuclear solvation, the equilibrium solvent reorganization energy of ET, $\lambda_{\mathrm{s}}$, and the solvent nuclear component of the equilibrium energy gap, $\Delta G_{\mathrm{s}}$, are multiplied with a nonergodicity function ${ }^{34} f\left(k_{\mathrm{ET}}, T\right)$, where the dependence on $T$ is produced mainly by the temperature-dependent relaxation time $\tau(T)$ of a mode driving electronic transition. The nonergodicity function thus reflects the fraction of the phase space the ET system is allowed to sample on the time window of the reaction $\tau_{\mathrm{ET}}=k_{\mathrm{ET}}{ }^{-1}$. The nonergodic reorganization energy and free energy gap thus become

$$
\begin{aligned}
\lambda\left(k_{\mathrm{ET}}, T\right) & =f\left(k_{\mathrm{ET}}, T\right) \lambda_{\mathrm{s}}(T) \\
\Delta G\left(k_{\mathrm{ET}}, T\right) & =f\left(k_{\mathrm{ET}}, T\right) \Delta G_{\mathrm{s}}(T)
\end{aligned}
$$

The activation free energy barrier $G^{\text {act }}$ formed by the nonergodic solvation energies in eq 3 becomes a function of the ET rate. Therefore, the solution for the rate constant requires solving a self-consistent equation ${ }^{34}$

$$
k_{\mathrm{ET}} \propto \exp \left[-\beta \Delta G^{\mathrm{act}}\left(k_{\mathrm{ET}}\right)\right]
$$

where $\beta=1 / k_{\mathrm{B}} T$.

The typical form of the nonergodicity function for liquid crystalline solvents is shown in Figure 2. It has been obtained by using experimental data for 7CB nematogen (dielectric constant, refractive index, and density ${ }^{25}$ ) at different, temperature-independent time windows $\tau_{\mathrm{ET}}$. The gap in the lines around the IN transition reflects the absence of experimental data in that range of temperatures. For slow reactions $\left(k_{\mathrm{ET}}<10^{7} \mathrm{~s}^{-1}\right)$, the reaction is ergodic in the isotropic phase, i.e., follows the transition-state description, but it loses ergodicity in the nematic phase (upper lines in Figure 2). With increasing the rate, the longitudinal relaxation time $\tau_{\mathrm{L}}(T)$ of the polar nematic crosses the ET time $\tau_{\mathrm{ET}}$, and the nonergodicity function starts to deviate from unity in both the nematic and isotropic phases. This is the regime of dynamical solvent arrest when the nuclear solvent mode coupled to ET cannot relax on the time scale of the reaction. ${ }^{34}$ Figure 2 indicates that, unless the reaction rate falls below $10^{8} \mathrm{~s}^{-1}$, the solvation component of the activation barrier is noticeably temperature dependent, even in the isotropic phase, because of nonergodicity. Therefore, any Arrhenius analysis neglecting these effects cannot be consistent with experiment, as indeed follows from the analysis of temperature-dependent ET rates in the isotropic phase of liquid crystalline solvents. ${ }^{12}$
The effect of the dynamical arrest of nuclear solvation on the reaction rate is easiest to understand for charge-separation reactions. In this case, the vertical energy gap for electronic transition, $\Delta E=\lambda_{\mathrm{i}}+\lambda_{\mathrm{s}}+\Delta G_{\mathrm{CS}}$ ( $\lambda_{\mathrm{i}}$ is the classical intramolecular reorganization energy and $\Delta G_{\mathrm{CS}}$ is the free energy gap for charge separation), depends on electronic solvation only and changes little with lowering temperature. The activation barrier

$$
\Delta G^{\mathrm{act}}\left(k_{\mathrm{ET}}, T\right)=\frac{\Delta E^{2}}{4\left(\lambda\left(k_{\mathrm{ET}}, T\right)+\lambda_{\mathrm{i}}\right)}
$$

will then increase in the nonergodic region due to the lowering of $\lambda\left(k_{\mathrm{ET}}, T\right)$, and charge separation will slow. The rate of charge separation in a slowly relaxing nematic solvent will be slower than the rate in a fast-relaxing isotropic solvent with comparable polarity, as indeed observed in experiment. ${ }^{9-12,14}$

In Section II below, we will formulate a theoretical description of the nonergodic solvation energy in nematics. The complete description of the solvent effect on ET requires accounting for such factors as orientation of the donor-acceptor complex relative to the nematic director and the possibility of different orientations of the solvent dipole relative to the long rotational axis of the liquid crystalline molecules. This, relatively complete, description of the problem is achieved at the expense of a simplified geometry and charge distribution of the solute approximated by a point dipole. A more complete description of the solute geometry and charge distribution in terms of atomic coordinates and charges can be achieved in the isotropic phase of the solvent. This latter approach is applied to the analysis of experimental data from Wasielewski's group on ET in a donoracceptor molecule dissolved in the isotropic phase of 4-pentyl4-cyanobiphenyl (5CB) liquid crystal (Section III). Finally, we conclude in Section IV.

\section{Solvation in Nematics}

To define the nonergodicity function in eq 3 , we will follow the common arguments from the linear response theory. ${ }^{46}$ If charge separation at time $t=0$ produces the charge-transfer dipole $\Delta m_{0}$, the following relaxation of the solvent creates the nuclear reaction field $R(t)$, which can be obtained from the causality relation with the response function $\chi\left(t-t^{\prime}\right)$ :

$$
R(t)=\int_{-\infty}^{t} \chi\left(t-t^{\prime}\right) \Delta m_{0}\left(t^{\prime}\right) \mathrm{d} t^{\prime}
$$

The free energy of equilibrium solvation can then be obtained from the correlation function of the reaction field fluctuations

$$
\Delta G_{\mathrm{s}}=-\frac{1}{2} \beta\left(\Delta m_{0}\right)^{2}\left\langle(\delta R)^{2}\right\rangle
$$

The equilibrium autocorrelation function $\left\langle(\delta R)^{2}\right\rangle$ in this equation is related, through the fluctuation-dissipation theorem, to the imaginary part $\chi^{\prime \prime}(\omega)$ of the Fourier transform of $\chi(t)$ as follows ${ }^{46}$

$$
\Delta G_{\mathrm{s}}=-\left(\Delta m_{0}\right)^{2} \int_{0}^{\infty} \chi^{\prime \prime}(\omega)(\mathrm{d} \omega / \pi \omega)
$$

Following the common arguments of linear theories of solvation dynamics, ${ }^{47-49} \chi(\omega)$ can be obtained by using the frequencydependent solvent parameters affecting the response function. In particular, when solvent polarity is defined through the solvent dielectric constant $\epsilon(\omega)$, the response function $\chi(\omega)$ depends on frequency through the dielectric constant: ${ }^{47}$

$$
\chi(\omega)=\chi[\epsilon(\omega)]
$$


The integral over all frequencies of various solvent modes in eq 8 defines the equilibrium, ergodic solvation free energy. The slow modes, characterized by frequencies below the rate of transition, cannot relax on the time scale of the reaction and should be eliminated from the frequency integral in order to obtain nonergodic $\Delta G\left(k_{\mathrm{ET}}, T\right)$. The simple stepwise frequency filter gives results in a good agreement with the direct average of the energy fluctuations on a given observation window. ${ }^{41,50}$ The nonergodic solvation free energy in eq 3 becomes

$$
\Delta G\left(k_{\mathrm{ET}}, T\right)=-\left(\Delta m_{0}\right)^{2} \int_{k_{\mathrm{ET}}}^{\infty} \chi^{\prime \prime}(\omega)(\mathrm{d} \omega / \pi \omega)
$$

In the case of solvation of an ion in an isotropic dielectric, $\chi(\omega)=(4 \pi)^{-1}\left(1 / \epsilon_{\infty}-1 / \epsilon(\omega)\right)$, where $\epsilon_{\infty}$ is the high-frequency dielectric constant. When Debye dielectric relaxation is used for $\epsilon(\omega)$, one gets for $f\left(k_{\mathrm{ET}}, T\right)$ in eqs 3 and 10

$$
f\left(k_{\mathrm{ET}}, T\right)=(2 / \pi) \operatorname{arccot}\left(k_{\mathrm{ET}} \tau_{\mathrm{L}}(T)\right)
$$

where $\tau_{\mathrm{L}}(T)$ is the longitudinal dielectric relaxation time. The function $f\left(k_{\mathrm{ET}}, T\right)$ in eq 11 normally has a sigmoidal form dropping from its equilibrium value $f=1$ at high temperatures to lower, nonergodic values once the reaction time crosses the longitudinal dielectric relaxation time. The nonergodicity function shown in Figure 2 has been obtained from the solution of the solvation problem in a more complex, dipolar geometry of the solute with the account of the axial symmetry of the liquid crystal and the microscopic nature of the solvent response (see below).

The approximation of the solvent by a continuum dielectric is not reliable when reactions in liquid crystals are studied. The importance of correlations between molecular dipoles becomes clear from considering the inverted-space representation of the autocorrelation function of dipolar polarization. An analogue of the density structure factor in atomic liquids, this correlation function is a second-rank tensor ${ }^{46}$

$$
S_{\alpha \beta}(\mathbf{k})=\frac{3}{N}\left\langle\sum_{i j} \hat{e}_{i, \alpha} \hat{e}_{j, \beta} e^{-i \mathbf{k} \cdot r_{i j}}\right\rangle
$$

where $\hat{e}_{i, \alpha}=m_{i, \alpha} / m$ is the Cartesian projection of the unit vector in the direction of the dipole moment $\mathbf{m}_{i}$ in the laboratory system of coordinates. In the same system of coordinates, the position of the point dipole at solvent molecule $i$ is defined by $\mathbf{r}_{i}$, the sum in eq 12 runs over the $N$ solvent molecules, and $\mathbf{k}$ is the wave vector.

If the $z$-axis of the coordinate system is chosen along the nematic director $\hat{\mathbf{n}}$, two diagonal components of the tensor $S_{\alpha \beta^{-}}$ (k), $S_{z z}(\mathbf{k})$ and $S_{x x}(\mathbf{k})=S_{y y}(\mathbf{k})$, are most significant. ${ }^{52}$ Because of the axial symmetry of the solvent, $S_{\alpha \beta}(\mathbf{k})$ can be expanded in Legendre polynomials of $\hat{\mathbf{k}} \cdot \hat{\mathbf{n}}$ :

$$
S_{\alpha \beta}(\mathbf{k})=\sum_{l} S_{\alpha \beta, l}(k) P_{1}(\hat{\mathbf{k}} \cdot \hat{\mathbf{n}})
$$

where $\hat{\mathbf{k}}=\mathbf{k} / k$. Angular projections $S_{\alpha \beta, l}(k)$ calculated from computer simulations ${ }^{52}$ show that the $l=0$ component is the main contributor to the tensor $S_{\alpha \beta}(\mathbf{k})$. Therefore, for most practical applications, the angular average can be used

$$
S_{\alpha \beta}(k)=\left\langle S_{\alpha \beta}(\mathbf{k})\right\rangle_{\omega_{k}}
$$

where $\langle\cdots\rangle_{\omega_{k}}$ stands for the average over the orientations of $\hat{\mathbf{k}}$.

Figure 3 shows the diagonal projections $S_{z z}(k)$ and $S_{x x}(k)$ obtained from Monte Carlo (MC) simulations of a fluid of hard

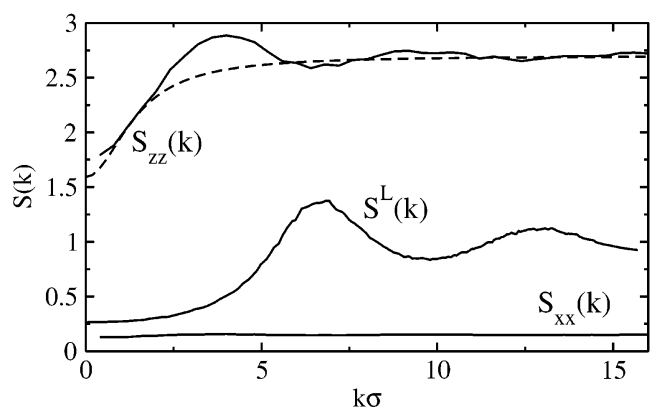

Figure 3. Dipolar structure factors $S_{z z}(k)$ and $S_{x x}(k)$ of a fluid of spherocylinders with centered dipole moment ${ }^{31}$ and the longitudinal structure factor of a fluid of hard-sphere dipoles $S^{\mathrm{L}}(k) .{ }^{51}$ The reduced dipole moment $\beta \mathrm{m}^{2} / \sigma^{3}$ is equal to 1.0 for both fluids. The aspect ratio for spherocylinders is $L / \sigma=5.0$. The packing fraction is $\eta=(\pi / 6) \rho \sigma^{3}$ $=0.419$ for hard spheres and $\eta=(\pi / 6) \rho \sigma^{3}(1+3 L / 2 \sigma)=0.470$ for spherocylinders. The dashed line shows the Padé approximation of eq 17.

spherocylinders ${ }^{31}$ (aspect ratio 5) with centered point dipoles. These correlation functions are compared with the corresponding function for an isotropic fluid of dipolar hard spheres. In the latter case, the longitudinal projection on the wave vector, most relevant for solvation studies ${ }^{51}\left(S^{\mathrm{L}}(k)\right.$, see eq 27 below), is taken. The dipolar projections of structure factors obtained from simulations of force field molecular liquids ${ }^{53,54}$ share all basic features with this model fluid. The structure factor $S^{\mathrm{L}}(k)$ of isotropic dipolar solvents shows a plateau at $k \sigma<\pi$. Because, for large solutes with the effective radius $R_{0} \gg \sigma$, the range of $k$ values affecting solvation is $k<\pi / R_{0}$, the approximation $S^{\mathrm{L}_{-}}$ $(k) \simeq S^{\mathrm{L}}(0)$, resulting in continuum solvation, is reasonably accurate (deviations from this approximation are responsible for observable effects on the solvation entropy $\left.{ }^{54,55}\right)$. The perpendicular component $S_{x x}(k)$ in liquid crystals is governed by repulsions of long molecular rods at the distance of shortaxis diameter $\simeq \sigma$ and is, therefore, qualitatively similar to $S^{\mathrm{L}_{-}}$ $(k)$ in isotropic solvents.

The correlation of dipoles in the direction parallel to the director is determined by the long dimension $L$ of the molecular rods making up the liquid crystal. This larger length scale is reflected by a much steeper rise of $S_{z z}(k)$ to its $k \rightarrow \infty$ plateau. An additional factor contributing to a steeper rise of $S_{z z}(k)$ is the level of the plateau. The values of $S_{z z}(\infty)$ and $S_{x x}(\infty)$ are determined by the order parameter $S_{2}$ and the angle $\phi$ between the direction of the permanent dipole and the long molecular axis of the solvent molecule (Figure 4):

$$
\begin{aligned}
& S_{z z}(\infty)=1+2 S_{2} P_{2}(\cos \phi) \\
& S_{x x}(\infty)=1-S_{2} P_{2}(\cos \phi)
\end{aligned}
$$

In eq 15 , the order parameter $S_{2}$ is an ensemble average of the second Legendre polynomial of the dot product between the unit vector $\hat{\mathbf{l}}$ along the long rotational axis of the nematic molecule and the director (Figure 4):

$$
S_{2}=\left\langle P_{2}(\hat{\mathbf{l}} \cdot \hat{\mathbf{n}})\right\rangle
$$

Perfect alignment results in $S_{2}=1$, while $S_{2} \simeq 0.5-0.7$ is typically observed in real nematics ${ }^{29}$ (Figure $1 \mathrm{~b}$ ). For nematics with positive anisotropy, $\phi$ is small and $\epsilon_{\|}>\epsilon_{\perp}$ (Figure 1a). In this case, the high level of the $k \rightarrow \infty$ plateau $\left(S_{z z} \simeq 1+2 S_{2}\right.$ in eq 15) contributes to a faster rise of $S_{z z}(k)$, as is shown in Figure 3 for the case of purely longitudinal dipoles $(\phi=0){ }^{31}$

Once the size of the solute does not significantly exceed the long dimension $L$ of the nematic molecules, the continuum limit 


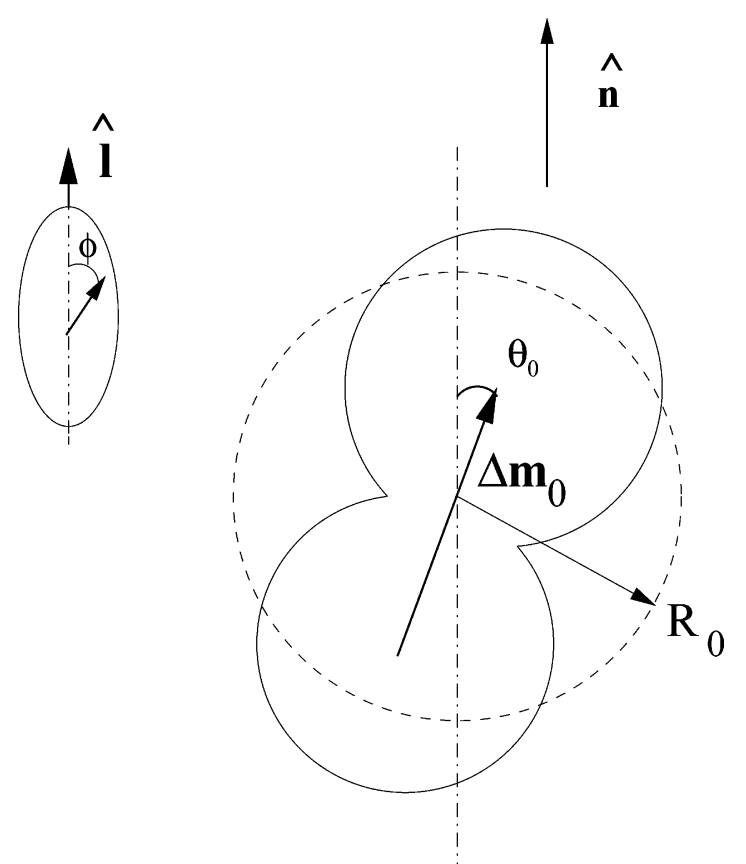

Figure 4. Diagram of a dipolar solute in a nematic solvent. The dashed line shows the representation of a donor-acceptor dyad by a sphere of radius $R_{0}$. The angle $\theta_{0}$ is between the charge-transfer dipole $\Delta \mathbf{m}_{0}$ and the nematic director $\hat{\mathbf{n}}$, and the angle $\phi$ is between the solvent dipole and the long rotation axis of the solvent molecule given by the unit vector $\hat{\mathbf{l}}$.

for solvation, $S_{\alpha \beta}(k) \simeq S_{\alpha \beta}(0)$, is not reliable, and one needs to take into account the nonlocal response of the dipoles in the liquid crystal. This can be accomplished by approximating the dipolar correlation function between its $k=0$ and $k \rightarrow \infty$ limits by a Padé form ${ }^{56}$ (dashed line in Figure 3 )

$$
S_{\alpha \beta}(k)=\frac{S_{\alpha \beta}(0)+S_{\alpha \beta}(\infty) \Lambda^{2} k^{2}}{1+\Lambda^{2} k^{2}}
$$

This equation introduces a new theory parameter, the dipolar correlation length $\Lambda$ (assumed to be isotropic, see ref 52 for discussion). Note that $\Lambda$ is the correlation length of the firstrank tensor of the dipolar polarization, in contrast to the correlation length $\xi_{\mathrm{Q}}$ of the second-rank tensor of the nematic order parameter. Computer simulations indicate that $\Lambda$ is continuous through the first-order IN transition, ${ }^{31,52}$ whereas $\xi_{\mathrm{Q}}$ is expected to show pretransitional critical divergence. ${ }^{21}$

The approximation of eq 17 allows us to obtain a closedform solution for the solvation response function of a spherical dipolar solute in a solvent with axial symmetry ${ }^{52}$

$$
\begin{aligned}
\chi(\omega)=\chi_{\mathrm{c}}(\omega)+\left(\chi_{\infty}-\chi_{\mathrm{c}}(\omega)\right) \frac{3 \Lambda}{2 R_{1}} \times & \\
& {\left[1-\left(\frac{\Lambda}{R_{1}}\right)^{2}+\left(1+\frac{\Lambda}{R_{1}}\right)^{2} e^{-2 \frac{R_{1}}{\Lambda}}\right] }
\end{aligned}
$$

where $R_{1}=R_{0}+\sigma / 2$ is the distance of the closest approach of a solvent dipole to the solute of the radius $R_{0}$. In eq $18, \chi_{\mathrm{c}}(\omega)$ is the continuum response function corresponding to the $k=0$ limit in the polarization correlation functions of the solvent. It can be obtained by solving the Poisson equation for solvation of a dipole in an axially symmetric solvent: ${ }^{.7}$

$$
\chi_{\mathrm{c}}(\omega)=\frac{1}{2 R_{1}^{3}}\left(R_{x}(\omega)+\left(R_{z}(\omega)-R_{x}(\omega)\right) \cos ^{2} \theta_{0}\right)
$$

where $\theta_{0}$ is the angle between the solute dipole and the director (Figure 4).

The anisotropic reaction field of the solvent is given by parallel, $R_{z}$, and perpendicular, $R_{x}$, projections in respect to the nematic director:

$$
\begin{aligned}
& R_{z}(\omega)=\frac{\epsilon_{\perp}(\omega)+2-\left(\epsilon_{\|}(\omega)+2\right) \psi(\omega)}{\epsilon_{\perp}(\omega)-1-\left(\epsilon_{\|}(\omega)-1\right) \psi(\omega)} \\
& R_{x}(\omega)= \\
& \frac{\epsilon_{\perp}(\omega)\left(2 \epsilon_{||}(\omega)-\epsilon_{\perp}(\omega)+2\right)-\epsilon_{||}(\omega)\left(\epsilon_{\perp}(\omega)+2\right) \psi(\omega)}{\epsilon_{\perp}(\omega)\left(2 \epsilon_{\|}(\omega)-\epsilon_{\perp}(\omega)-1\right)-\epsilon_{\|}(\omega)\left(\epsilon_{\perp}(\omega)-1\right) \psi(\omega)}
\end{aligned}
$$

Here,

$$
\psi(\omega)=\int_{0}^{1} \frac{\mathrm{d} z}{1+\left(\epsilon_{||}(\omega) / \epsilon_{\perp}(\omega)-1\right) z^{2}}
$$

The deviation of $\chi(\omega)$ from the continuum limit $\chi_{\mathrm{c}}(\omega)$ in eq 18 is caused by the nonlocal nature of the dipolar correlation functions (dependence on $k$ ). The magnitude of this deviation is determined by the polarization correlation length $\Lambda$ and the value of the response function $\chi_{\infty}$ obtained from the $k \rightarrow \infty$ limit of $S_{\alpha \beta}(k)$. It is given by the following relation

$$
\chi_{\infty}=\frac{y}{R_{1}^{3}}\left(1+\frac{1}{5} S_{2} P_{2}(\cos \phi) P_{2}\left(\cos \theta_{0}\right)\right)
$$

where $y=(4 \pi / 9) \beta m^{2} \rho$, and $\rho$ and $m$ are, respectively, the number density and dipole moment of the nematic solvent.

The calculations shown in Figure 2 are done by using $\chi(\omega)$ from eq 18 with the dielectric function $\epsilon(\omega)$ of 7CB measured experimentally. ${ }^{20}$ The experimental data are well represented by the Debye dielectric function with the temperature-dependent relaxation time shown in Figure 1c. Two observations are relevant to our discussion. First, there is a weak discontinuity of the nonergodicity function at $T_{\mathrm{IN}}$. Second, the nonergodicity function is only weakly anisotropic in the nematic phase. Both of these results are a consequence of the independence of the correlation length $\Lambda$ in eq 18 of frequency. As a result of this assumption, the function $\chi^{\prime \prime}(\omega) / \chi^{\prime \prime}(0)$ is fully determined by its continuum limit, which shows little anisotropy of solvation energies in the nematic phase.

In contrast to the nonergodicity function, which is mostly determined by continuum dielectric response, the equilibrium free energy of solvation is sensitive to microscopic properties of dipolar solvent correlations. Figure 5 compares the microscopic and continuum calculations of the equilibrium free energy (equal to half the Stokes shift) of solvation of the solute dipole $\Delta m_{0}$. The difference between the two results comes from the steep rise of the dipolar structure factor $S_{z z}(k)$ neglected in the continuum limit $S_{z z}(k)=S_{z z}(0)$. As a result, the absolute value of the solvation energy is significantly larger than its continuum limit, and the splitting between the energies calculated for the parallel and perpendicular orientations of the solute dipole is significantly wider. We need to note that continuum calculations commonly fall below the microscopic result when the distance of the closest approach $R_{1}$ is used for the solute size in both methods. ${ }^{51}$ This outcome is normally offset in continuum applications by choosing the solute radius equal to the van der Waals radius $R_{0}$. For the data given in Figure 5 , the radius change multiplies the continuum result by a factor of 3.6. The continuum calculation in the isotropic phase then falls close to the microscopic result (dash-dotted line in the upper panel in 


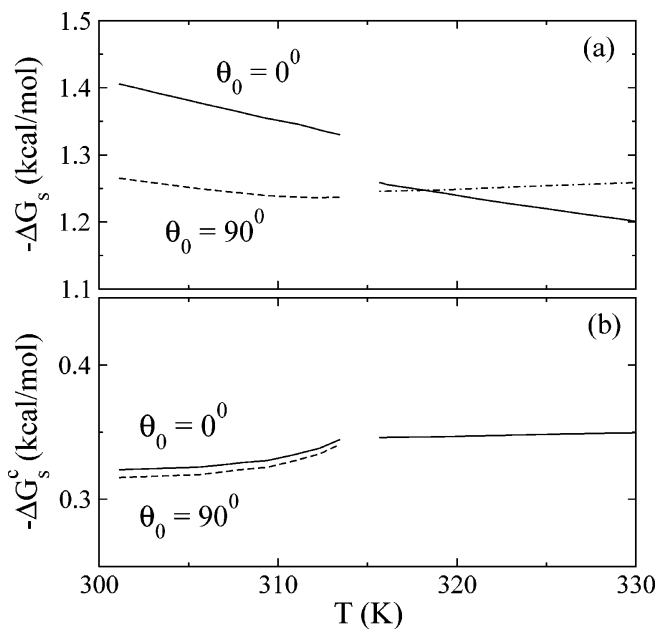

Figure 5. Temperature dependence of the solvation free energy calculated from the microscopic level of theory (a, eqs 8 and 18) and from the continuum limit (b, eqs 8 and 19). The solute radius and dipole moment are $R_{0}=4.9 \AA$ and $\Delta m_{0}=7.5 \mathrm{D}$, respectively (parameters for the Stokes shift in the coumarin-153 optical dye, see ref 52). The solvent parameters are those of 7CB with the dipole moment $4.347 \mathrm{D}$, $\sigma=5.27 \AA, \phi=27^{\circ}$ (Figure 4), and the dipolar correlation length $\Lambda$ $=0.45 \sigma$ obtained from Monte Carlo simulations of a fluid of dipolar spherocylinders. ${ }^{52}$ The dash-dotted line in the upper panel is the continuum isotropic result calculated with the van der Waals radius $R_{0}$ for the dielectric cavity.

Figure 5). The slope of the continuum free energy is, however, incorrect, as is often the case with continuum calculations of the solvation entropy. ${ }^{54-56,58}$

\section{Comparison to Experiment. Isotropic Phase}

The calculations presented above indicate that transition from the isotropic to nematic phase does not significantly affect the nonergodicity function, except for weak discontinuities at the transition temperature and relatively weak anisotropy with respect to the solute's orientation relative to the nematic director. For subnanosecond reactions, dynamical arrest of solvent reorganization is fully present already in the isotropic phase. This observation allows us to study this phenomenon, avoiding complications related to the global axial symmetry of the solvent in the nematic phase. The calculations presented below have been done for the donor-acceptor complex (Figure 6) extensively studied by Wasielewski's group. ${ }^{10,11}$ Following refs 10 and 11 , this donor-acceptor molecule, 4-( $N$-pyrrolidino)naphthalene-1,8-imide-pyromellitimide, will be referred to as PNI-PI.

A. Nonergodic Reorganization Energy. Complications with two symmetry axes (wave vector $\hat{\mathbf{k}}$ and the nematic director $\hat{\mathbf{n}}$ ) in the nematic phase can be avoided in the isotropic phase, at least for the macroscopically observable properties. Locally, a donor-acceptor complex may be affected by the anisotropic environment of a nematic domain, although solvation dynamics studies suggest that probe molecules reside in the part of the solvent between the domains. ${ }^{59-61}$ Because there are no experimentally available parameters characterizing this local environment, we will assume that the solvent (5CB) is an isotropic liquid with the dielectric parameters provided by dielectric measurements. ${ }^{27,62}$ Within this approximation, solvation thermodynamics and dynamics can be treated on a rather sophisticated level with atomic resolution of the solute structure and charge distribution as well as with the full nonlocal response of the solvent dipolar polarization. ${ }^{63}$

A microscopic theory of polar solvation dynamics has been recently proposed by one of us. ${ }^{63}$ The theory calculates the Laplace transform of the solute-solvent interaction energy $E(s)$ appearing as a linear response to switching on the solute electric field $\mathbf{E}_{0}(\mathbf{r})$. In the linear response approximation, the solution is given in terms of the solvation response function $\chi(\mathbf{k}, s)$ in wave vector-Laplace space $\{\mathbf{k}, s\}$ :

$$
E(s)=-s^{-1} \tilde{\mathbf{E}}_{0}(\mathbf{k}) * \chi(\mathbf{k}, s) * \tilde{\mathbf{E}}_{0}(-\mathbf{k})
$$

Here, $\tilde{\mathbf{E}}_{0}(\mathbf{k})$ is the Fourier transform of the solute field taken over the volume outside the repulsive core of the solute, including the addition of the solvent radius to the solute atoms exposed to the solvent $\left(R_{1}\right.$ vs $R_{0}$ for a spherical solute in Section II). The stars in eq 23 denote a 3D integral in $\mathbf{k}$-space and tensor contraction over the Cartesian indexes. The Laplace transform is preferable for this formulation because it allows us to use real frequency-dependent dielectric constants. The function

$$
F(s)=-s E(s)
$$

then directly leads to the nonergodic reorganization energy by analytic continuation to complex frequencies:

$$
\lambda\left(k_{\mathrm{ET}}, T\right)=\int_{k_{\mathrm{ET}}}^{\infty} F^{\prime \prime}(-i \omega)(\mathrm{d} \omega / \pi \omega)
$$

where $F^{\prime \prime}(z)$ denotes the imaginary part of $F(z)$.

The linear solvation response function $\chi(\mathbf{k}, s)$ is calculated according to the formalism outlined in ref 63 . In short, this procedure provides a connection between $\chi(\mathbf{k}, s)$ and the dipolar susceptibility of the polar solvent $\chi_{\mathrm{s}}(\mathbf{k}, s)$ for a solute of an arbitrary shape and charge distribution. Inverted space for isotropic liquids is characterized by axial symmetry set up by the wave vector. The second-rank tensor of solvent susceptibility is then fully characterized by its two projections, longitudinal (L) and transverse $(\mathrm{T}):^{48,63}$

$$
\begin{aligned}
\chi_{\mathrm{s}}^{\mathrm{L}, \mathrm{T}}(k, s)=\chi_{\mathrm{s}}^{\mathrm{L}, \mathrm{T}}(0,0)\left[\frac{S^{\mathrm{L}, \mathrm{T}}(0)}{S^{\mathrm{L}, \mathrm{T}}(k)}+\right. & \\
& \left.\frac{1}{1+p^{\prime}(k \sigma)^{2}} \frac{\chi_{\mathrm{s}}^{\mathrm{L}, \mathrm{T}}(0,0)-\chi_{\mathrm{s}}^{\mathrm{L}, \mathrm{T}}(0, s)}{\chi_{\mathrm{s}}^{\mathrm{L}, \mathrm{T}}(0, s)}\right]^{-1}
\end{aligned}
$$

In eq $26, S^{\mathrm{L}, \mathrm{T}}(k)$ are the structure factors of the solvent dipolar

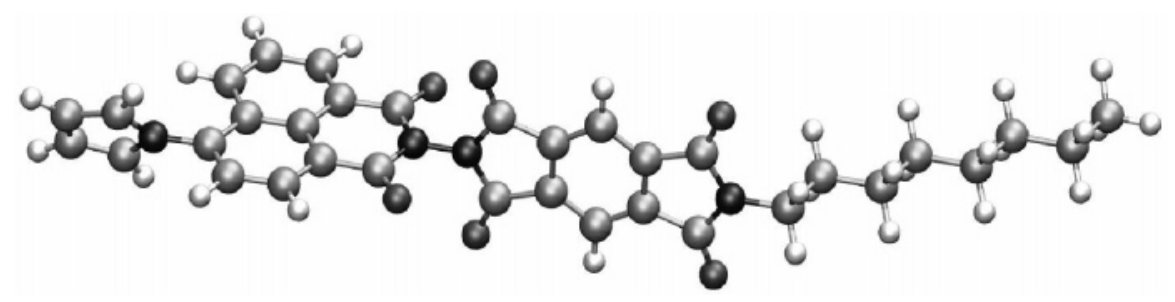

Figure 6. Donor-acceptor molecule, PNI-PI, studied in refs 10 and 11. 
TABLE 1: Parameters Used to Calculate the Charge-Separation (CS) and Charge-Recombination (CR) Rates in PNI-NI (Figure 6) from Eqs 32-37

\begin{tabular}{ccccccccc}
\hline reaction & $V_{\mathrm{ET}}, \mathrm{cm}^{-1}$ & $\omega_{\mathrm{v}}, \mathrm{cm}^{-1}$ & $\Delta G_{\mathrm{CS}}^{\prime}, \mathrm{eV}$ & $\lambda_{i}, \mathrm{eV}$ & $\lambda_{v}, \mathrm{eV}$ & $a, \mathrm{eV}$ & $b, \mathrm{eV} / \mathrm{K}$ & $c, \mathrm{eV} / \mathrm{K}^{2}$ \\
\hline $\mathrm{CS}+\mathrm{CR}$ & 144 & 1400 & 0.13 & 0.5 & 0.3 & 0.864 & $1.595 \times 10^{-3}$ & $1.2491 \times 10^{-6}$
\end{tabular}

${ }^{a}$ Parameters $V_{\mathrm{ET}}, \lambda_{i}$, and $\Delta G_{\mathrm{CS}}^{\prime}$ are obtained from the simultaneous fit of CS and CR experimental rates shown in Figure 8. Equilibrium reorganization energy $\lambda_{\mathrm{s}}(T)$ is calculated as described in Section IIIA, and its temperature dependence is given by a bilinear regression: $\lambda_{\mathrm{s}}(T)=a$ $-b \times T+c \times T^{2}$.

polarization. ${ }^{51,64}$ These follow from eq 12 by taking the longitudinal and transverse projections

$$
\begin{aligned}
S^{\mathrm{L}}(k) & =\sum_{\alpha, \beta} \hat{k}_{\alpha} \hat{k}_{\beta} S_{\alpha, \beta}(k) \\
S^{\mathrm{T}}(k) & =\frac{1}{2}\left(\sum_{\alpha} S_{\alpha, \alpha}(k)-S^{\mathrm{L}}(k)\right)
\end{aligned}
$$

Finally, the susceptibilities $\chi_{\mathrm{s}}^{\mathrm{L}, \mathrm{T}}(0,0)$ at $\{k, s\}=0$ in eq 26 are expressed in terms of the static, $\epsilon_{\mathrm{s}}=\epsilon_{\mathrm{s}}(0)$, and high-frequency, $\epsilon_{\infty}$, dielectric constants; ${ }^{63} \chi_{\mathrm{s}}(0, s)$ are defined in terms of the frequency-dependent dielectric constant $\epsilon_{\mathrm{S}}(s)$.

The parameter $p^{\prime}$ in eq 26 is proportional to the ratio of the translational and rotational diffusion coefficients in the liquid. It quantifies the effect of translational diffusion on polarization dynamics. ${ }^{65}$ This effect is expected to be small in liquid crystalline solvents and is neglected in the present calculations $\left(p^{\prime}=0\right)$. The structure factors of dipolar polarization $S^{\mathrm{L}, \mathrm{T}}(k)$ are calculated according to the formalism based on the meanspherical approximation for dipolar liquids ${ }^{66}$ reparametrized to fit the results of simulations. ${ }^{51}$

The structure and charge distribution of PNI-PI (Figure 6) in the neutral ground state was obtained from RHF/6-31G calculations using GAUSSIAN 03.67 The atomic coordinates and charges, obtained from CHELPG method, are used as input to define the solute repulsive core and the electric field. OPLS parametrization ${ }^{68}$ is used for the atomic van der Waals radii. The radius $\sigma / 2(\sigma=5.27 \AA)$ along the shorter axis of $5 \mathrm{CB}$ is added to the solute atoms exposed to the solvent to account for the distance of the closest approach between the solute and the solvent molecules. The solute charges in the charge-separated state $\mathrm{D}^{+}-\mathrm{A}^{-}$were calculated for separate $\mathrm{D}^{+}$and $\mathrm{A}^{-}$fragments at the RHF/6-31G level from the geometry optimized for the whole PNI-PI molecule in the neutral D-A state. The bonds broken to produce $\mathrm{D}^{+}$and $\mathrm{A}^{-}$fragments were terminated with hydrogens.

The atomic coordinates in the ground neutral state define the geometry of the complex. The charges in the ground and excited states are used to calculate the difference charges $\Delta q_{j}(j=1$, ..., $M, M=72$ for PNI-PI) between the ground, $\mathrm{D}-\mathrm{A}$, and charge-separated, $\mathrm{D}^{+-} \mathrm{A}^{-}$, states. The corresponding difference electric field $\Delta \mathbf{E}_{0}(\mathbf{r})$, produced by charges $\Delta q_{j}$, is Fourier transformed and used instead of $\tilde{\mathbf{E}}_{0}(\mathbf{k})$ in eq 23 in order to calculate the Stokes shift correlation function and the nonergodic reorganization energy (eq 25). Assuming the Debye form for the dielectric constant

$$
\epsilon(s)=\epsilon_{\infty}+\frac{\epsilon_{\mathrm{s}}-\epsilon_{\infty}}{1+s \tau_{\mathrm{D}}}
$$

this calculation results in a simple single-exponential form for $F(s, T) / 2=\lambda_{\mathrm{s}}(T) /\left(a(T) s \tau_{\mathrm{D}}(T)+1\right)$, where $\tau_{\mathrm{D}}(T)$ is the Debye relaxation time of the solvent and the equilibrium solvent reorganization energy $\lambda_{\mathrm{s}}(T)$ is listed in Table 1 . In addition, the function $a(T)$ can be fitted to a second-order polynomial: $a(T)$ $=2.165-0.00907 \times T+1.209 \times 10^{-5} T^{2}$.

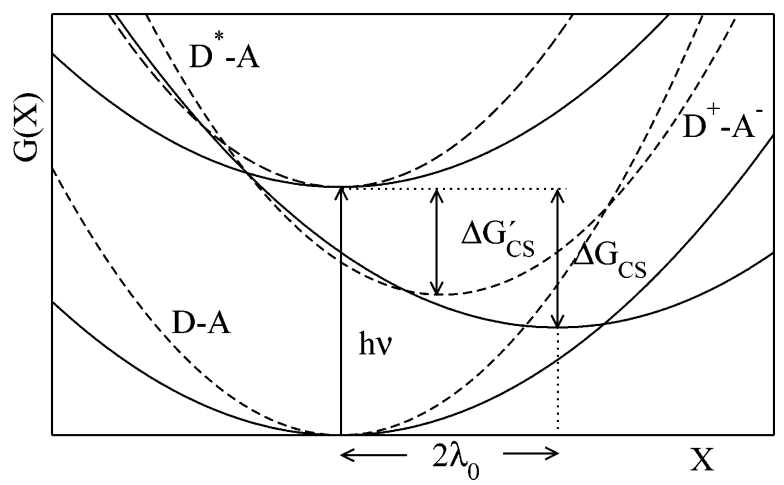

Figure 7. Diagram of the energy levels for photoexcited charge separation and charge recombination vs the reaction coordinate equal to the instantaneous energy gap between neutral and charge-transfer electronic states. The dashed lines refer to the fast reactions when the curvature of classical parabolas is $1 / 2 \lambda_{\mathrm{i}}$. The solid lines refer to slow reactions when full equilibration of the classical nuclear modes is achieved on the time scale of the reaction and the curvature becomes $1 / 2 \lambda_{0}, \lambda_{0}=\lambda_{\mathrm{i}}+\lambda_{\mathrm{s}}$. In the latter case, the equilibrium energy of chargeseparated state is shifted down relative to the dashed curve by the amount of solvation free energy $\lambda_{s}$ (eq 31). The distance between the surface minima is equal to twice the reorganization energy equilibrated on the time scale of the reaction. The photoexcitation energy of the donor unit is $h v$ (equal to $2.65 \mathrm{eV}$ for $\mathrm{PNI}-\mathrm{PI}^{10}$ ).

B. Electron-Transfer Rate. Three types of nuclear modes will be considered in modeling ET in liquid crystalline 5CB. Intramolecular quantum vibrations of the solute are represented by a single effective vibrational mode with the frequency $\hbar \omega_{\mathrm{v}}$ $=1400 \mathrm{~cm}^{-1}(\mathrm{C}=\mathrm{C}$ stretch $)$ and the reorganization energy $\lambda_{\mathrm{v}}$. The total classical, equilibrium reorganization energy

$$
\lambda_{0}=\lambda_{\mathrm{s}}+\lambda_{\mathrm{i}}
$$

is a combination of two types of modes. $\lambda_{\mathrm{i}}$ absorbs all classical nuclear modes that cannot become dynamically arrested on the time scale of the reaction. These typically would incorporate classical intramolecular modes of the donor-acceptor complex and any fast solvent modes not included in the polarization response function. The component $\lambda_{\mathrm{s}}$ represents dipolar polarization of the solvent, and it is this mode that is of main interest to us because it becomes dynamically arrested on the time scale of ET.

A diagram of the energy levels for photoinduced charge separation and recombination is shown in Figure 7. The distinction between the free energy surfaces shown by the dashed and solid lines reflects the difference between fast and slow ET kinetics. When the reaction is fast compared to longitudinal polarization relaxation, the dipolar reorganization energy is frozen on the reaction time scale, and the curvature of the classical parabola is $1 / 2 \lambda_{\mathrm{i}}$. Correspondingly, the free energy gaps for charge separation, $\Delta G^{\prime}{ }_{\mathrm{CS}}$, and charge recombination, $\Delta G^{\prime}$ RR , are obtained by subtracting the free energy of dipolar solvation from the equilibrium energy gaps $\Delta G_{\mathrm{CS}}$ and $\Delta G_{\mathrm{CR}}$ :

$$
\Delta G_{\mathrm{CS}, \mathrm{CR}}^{\prime}=\Delta G_{\mathrm{CS}, \mathrm{CR}} \mp \Delta G_{\mathrm{s}}
$$


Here, "-" corresponds to charge separation and "+" corresponds to charge recombination. When the dipole moment of the initial photoexcited state is much smaller than the dipole moment of the charge-transfer state, one gets

$$
\Delta G_{\mathrm{s}}=-\lambda_{\mathrm{s}}
$$

The (partial) free energy gaps $\Delta G_{\mathrm{CS} / \mathrm{CR}}^{\prime}$ accumulate the difference in gas-phase energies of the final and initial ET states and the energy of nonpolar solvation arising from induction and dispersion forces. These interaction potentials normally do not significantly contribute to the reorganization energy ${ }^{69}$ and, by virtue of fast response of electronic solvent polarization, cannot become dynamically arrested. In addition, $\Delta G_{\mathrm{CS} / \mathrm{CR}}^{\prime}$ incorporates fast nuclear modes of the solvent contributing to the reorganization energy $\lambda_{\mathrm{i}}$. The solid lines in Figure 7 show the ET free energy surfaces for slow reactions when dipolar polarization is ergodic on the reaction time scale and the curvature of the free energy parabolas is $1 / 2 \lambda_{0}$. Because dipolar polarization is fully equilibrated on this time scale, the equilibrium energy of the charge-transfer state is below the dashed curve by the amount of solvation energy $\lambda_{\mathrm{s}}$ (eq 31 ).

The free energy diagram shown in Figure 7 is used to calculate the rate constants for charge separation and charge recombination. The ET model includes three types of nuclear modes: two classical modes, solvent polarization and lowfrequency vibrations, and one quantum mode of intramolecular solute vibrations. This set of modes was argued by Barbara and co-workers ${ }^{18}$ to form the minimum set required to describe inverted-region transitions affected by the solvent dynamics. The model describing such transitions was formulated ${ }^{18}$ as a hybrid of Sumi-Marcus ${ }^{17}$ and Jortner-Bixon ${ }^{70}$ models.

The ET rate is calculated as the inverse of the average time of decay of population of the initial ET state ${ }^{17}$

$$
k_{\mathrm{ET}}^{-1}=\int_{0}^{\infty} \mathrm{d} t \int_{-\infty}^{\infty} \mathrm{d} X P\left(k_{\mathrm{ET}}, X, t\right)
$$

In eq 32 , the probability $P\left(k_{\mathrm{ET}}, X, t\right)$ of finding the system in the initial ET state at time $t$ is calculated from the FokkerPlanck equation with the sink $k\left(k_{\mathrm{ET}}, X\right)$ produced by the classical and quantum vibrations of the solute at a given polarization of the solvent

$$
\begin{aligned}
& \frac{\partial}{\partial t} P\left(k_{\mathrm{ET}}, X, t\right)=D_{X}\left(k_{\mathrm{ET}}\right) \frac{\partial^{2} P\left(k_{\mathrm{ET}}, X, t\right)}{\partial X^{2}}+ \\
& \beta D_{X}\left(k_{\mathrm{ET}}\right) \frac{\partial}{\partial X}\left[P\left(k_{\mathrm{ET}}, X, t\right) \frac{\partial V\left(k_{\mathrm{ET}}, X\right)}{\partial X}\right]-k\left(k_{\mathrm{ET}}, X\right) P\left(k_{\mathrm{ET}}, X, t\right)
\end{aligned}
$$

The diffusion coefficient is $D_{X}\left(k_{\mathrm{ET}}\right)=2 \lambda\left(k_{\mathrm{ET}}\right) /\left(\beta \tau_{\mathrm{L}}\right)$ and the reaction potential is $V\left(k_{\mathrm{ET}}, X\right)=X^{2} /\left(4 \lambda\left(k_{\mathrm{ET}}\right)\right)$, where here and below the dependence on $T$ is dropped from $\lambda\left(k_{\mathrm{ET}}, T\right)$ for brevity. The rate constant at each configuration of the solvent is given by the semiclassical equation ${ }^{70,71}$

$$
k\left(k_{\mathrm{ET}}, X\right)=\frac{2 \pi\left|V_{\mathrm{ET}}\right|^{2}}{\hbar} e^{-\mathrm{S}} \sum_{n=0}^{\infty} \frac{S^{n}}{n !} G_{n}\left(k_{\mathrm{ET}}, X\right)
$$

Here, $V_{\mathrm{ET}}$ is the electron-transfer matrix element and $S=$ $\lambda_{\mathrm{v}} / \hbar \omega_{\mathrm{v}}$ is the Huang-Rhys factor. Further, $G_{n}(X)$ is the Gaussian distribution of energies for a single vibronic transition

$$
G_{n}\left(k_{\mathrm{ET}}, X\right)=\left[4 \pi \lambda_{\mathrm{i}} / \beta\right]^{-1 / 2} \exp \left[-\beta \frac{\Delta E\left(k_{\mathrm{ET}}, n, X\right)^{2}}{4 \lambda_{\mathrm{i}}}\right]
$$

The vertical energy gap $\Delta E(n, X)$ involves $n$ quanta of intramolecular vibrational excitation of the final ET state. For a charge-separation vibronic transition, it becomes

$$
\Delta E_{\mathrm{CS}}(n, X)=\Delta G_{\mathrm{CS}}^{\prime}+\lambda_{\mathrm{i}}+n \hbar \omega_{\mathrm{v}}-X
$$

Equation 36 assumes that the electrostatic coupling of the solute with the solvent is negligible in the initial state of the donor-acceptor complex obtained by photoexcitation of the donor unit. Therefore, nuclear polar solvation does not affect the vertical energy gap for charge separation (cf. eq 5). For charge recombination, the initial highly polar state $\mathrm{D}^{+}-\mathrm{A}^{-}$is solvated and the vertical energy gap becomes

$$
\Delta E_{\mathrm{CR}}\left(k_{\mathrm{ET}}, n, X\right)=\Delta G_{\mathrm{CR}}^{\prime}+2 \lambda\left(k_{\mathrm{ET}}\right)+\lambda_{\mathrm{i}}+n \hbar \omega_{\mathrm{v}}-X
$$

Equation 34 excludes classical vibrational dynamics of the solute from consideration assuming that equilibration along the vibrational coordinate is sufficiently fast to establish equilibrium distribution at each instantaneous configuration of the solvent. This is a reasonable approximation given slow fluctuations of the solvent polarization in nematics. Equations 32-37 are solved numerically based on the generalized moment expansion. ${ }^{72}$ The diffusion coefficient, the reaction potential, and sink rate are all dependent on the rate of ET through the reorganization energy $\lambda\left(k_{\mathrm{ET}}\right)$, including nonergodicity corrections. Because $\lambda$ $\left(k_{\mathrm{ET}}\right)$ does not enter the vertical gap for charge separation, $\Delta E_{\mathrm{CS}^{-}}$ $(n, X)$ in eq 36 is independent of the rate. Because of the dependence of nuclear solvation on $k_{\mathrm{ET}}$, the reaction rate is calculated by iterations in eq 32 .

In principle, each reaction, charge separation and charge recombination, is characterized by its own set of gas-phase and solvation parameters $\left\{V_{\mathrm{ET}}, \lambda_{\mathrm{v}}, \omega_{\mathrm{v}}, \Delta G^{\prime}, \lambda_{\mathrm{i}}, \lambda_{\mathrm{s}}\right\}$. Essentially, all these parameters are known with significant uncertainty. For the solvent reorganization, the geometry of only the neutral ground state has been calculated, and these results are used to calculate $\lambda_{\mathrm{s}}(T)$ for each temperature according to eqs 23-25. To avoid too many fitting parameters, we have carried out a global fit of both the charge-separation and charge-recombination experimental rates with three fitting parameters: $V_{\mathrm{ET}}, \lambda_{\mathrm{i}}$, and $\Delta G^{\prime}{ }_{C S}$ (Figure 8 and Table 1). The charge-recombination gap $\Delta G^{\prime}{ }_{\mathrm{CR}}$ is fixed by the condition $-\Delta G_{\mathrm{CS}}^{\prime}-\Delta G_{\mathrm{CR}}^{\prime}=h v=$ $2.65 \mathrm{eV}^{10}$ (see Figure 7), and the solvent component of $\Delta G_{\mathrm{CS} / \mathrm{CR}}$ is calculated from eq 31 . The value of the vibrational reorganization energy used in the calculations, $\lambda_{\mathrm{v}}=0.3 \mathrm{eV}$, was taken from the spectroscopic experiment ${ }^{73}$ (Table 1). We have also done fitting with the nonergodicity function equal to unity (thermodynamic limit). The global minimum of the fit was found at $\Delta G_{\mathrm{CS}}=-1.7 \mathrm{eV}$, which is probably too low, ${ }^{10}$ and the charge separation rate was about 5 times smaller than the one observed in experiment.

The main purpose of fitting the experimental kinetic data is to show that, within the observation window set up by the ET rate, reorganization of the dipolar solvent polarization is strongly nonergodic. Figure 9 shows that the nonergodicity function is well below its thermodynamic limit $f=1$ in the whole range of temperatures studied by experiment. The solvent reorganization energy of charge separation then does not exceed $35 \%$ of its thermodynamic value. This result may help to explain 


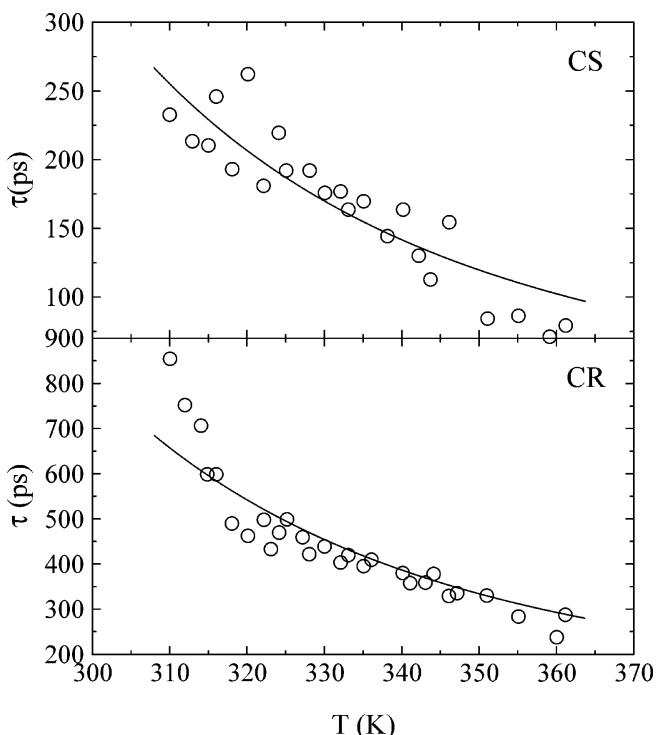

Figure 8. Experimental time of charge separation (CS, points) and charge recombination (CR, points) and the fit according to eq 32 (solid lines) in the isotropic phase of 5CB nematogen. The fit is obtained by varying $V_{\mathrm{ET}}, \Delta G_{\mathrm{CS}}^{\prime}$, and $\lambda_{\mathrm{i}}$ with the fitting parameters listed in Table 1.

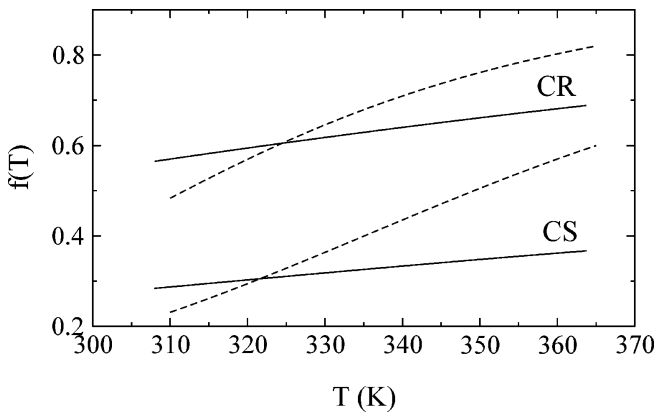

Figure 9. Nonergodicity function for charge separation (CS) and charge recombination (CR) for the isotropic phase of $5 \mathrm{CB}$ with the rate constants calculated from eq 32 and parameters obtained from the fit of experimental data (Table 1 and Figure 8). The dependence of the nonergodicity function on $k_{\mathrm{ET}}$ (eq 3 ) is dropped for the calculations represented by the solid lines because the rate constant is iteratively calculated at each temperature according to eqs $32-37$. The dashed line refer to the nonergodicity function calculated at the fixed ET rates of $5 \mathrm{~ns}^{-1}$ (CS) and $2 \mathrm{~ns}^{-1}$ (CR).

experimentally observed low sensitivity of ET to polarity of liquid crystals. ${ }^{74,75}$ The function $f(T)$ grows with increasing temperature due to faster solvent relaxation at higher temperatures. This trend is, however, offset by faster ET rates narrowing the observation window. When the observation window is fixed (dashed lines in Figure 9), the slope of $f(T)$ is greater, in qualitative agreement with the results shown in Figure 2.

Our current analysis does not reproduce the lowest-temperature points of the recombination time responsible for the nonArrhenius temperature dependence (Figure 8, lower panel). The use of the Zusman model for the solvent dynamic control of $\mathrm{ET}^{15}$ instead of the model by Barbara et al. ${ }^{18}$ actually gives the non-Arrhenius law due to a slight bend of the nonergodicity function at lowest temperatures. This observation suggests that further development of the model and/or the use of a narrower range of parameter values in the fit might improve the agreement with experiment.

We have also tested the effect of the temperature dependence of the energy gaps $\Delta G^{\prime}{ }_{\mathrm{CS} / \mathrm{CR}}$ on the fitting procedure. The free energy gaps $\Delta G^{\prime}{ }_{\mathrm{CS} / \mathrm{CR}}$ include the energy of induction solvation, which is proportional to the solvent density. ${ }^{41,69}$ The account

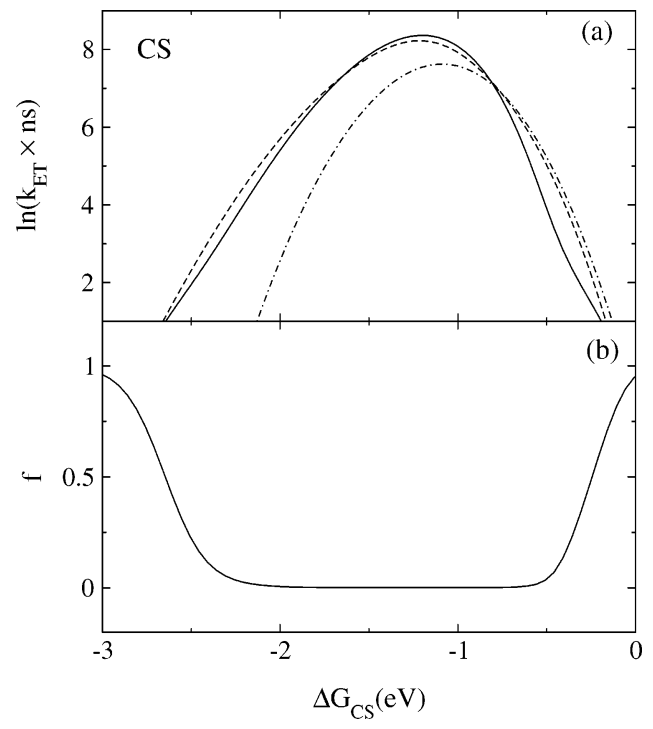

Figure 10. Rate constant of charge separation (a) and the nonergodicity function (b) vs the equilibrium free energy gap $\Delta G_{\mathrm{CS}}$ calculated for PNI-PI (Figure 6). The dash-dotted line in (a) shows the result of the nonadiabatic Bixon-Jortner model (eqs 38 and 39$)^{71}$ and the dashed line shows calculations with $f=1$, corresponding to the hybrid model of Barbara et al. ${ }^{18}$ The solid line in (a) refers to the self-consistent calculation according to eqs $32-37$ with iterative search for $k_{\mathrm{ET}}$ in eq 32 . The nonergodicity function in (b) is calculated from eqs 3 and 25 at $T=310 \mathrm{~K}$. The solute parameters are listed in Table 1 .

for the temperature variation of the density of $5 \mathrm{CB}$ does not significantly affect the results of the fit, although it makes the slopes of $f(T)$ higher by about $30 \%$. The overall nonergodic reorganization energy increases with temperature, in contrast to the equilibrium reorganization energy showing a negative temperature slope (Table 1).

The fact that dynamical arrest significantly reduces the solvent reorganization energy from its equilibrium value suggests that a part of the energy gap distribution is accommodated into a heterogeneous distribution of local environments of donoracceptor complexes. ${ }^{42,45}$ The impact of this heterogeneous distribution is expected to be diminished by the procedure used to prepare the initial state for charge separation. The distribution of energies of the initial locally excited (LE) state of the donoracceptor complex, created by the heterogeneous distribution of the local electric field, is proportional to the dipole moment of that state $m_{\mathrm{LE}}$. An additional reorganization energy arising from the heterogeneous distribution ${ }^{41}$ will be proportional to $m_{\mathrm{LE}}{ }^{2}$ and will be small compared to $\lambda_{0}$ when $m_{\mathrm{LE}} / \Delta m_{0} \ll 1$.

C. Energy Gap Law and Distance Dependence of ET Rates. Because the nonergodicity function is sensitive to the observation window, it seems reasonable to expect that changing the observation window at a given temperature may result in a sharp variation of the rate due to switching the reaction from a nonergodic to an ergodic regime. Such switching can be achieved by varying the ET energy gap. Figures 10 and 11 show the dependence of the rate of charge separation (Figure 10) and charge recombination (Figure 11) on the corresponding equilibrium free energy gaps, $\Delta G_{\mathrm{CS}}$ and $\Delta G_{\mathrm{CR}}$. In both cases, the nonergodicity function drops almost to zero in the range of energy gaps close to the top of the inverted parabola (fast reactions). When the reaction slows down, the nonergodicity function comes back to its equilibrium value $f \simeq 1$.

The calculations of charge separation rates are not very sensitive to the nonergodicity effect, as is seen from the comparison of the solid and dashed lines in Figure 10. Both calculations, ergodic and nonergodic, using the hybrid model in eqs $32-37$, arecompared to the nonadiabatic Bixon-Jortner 


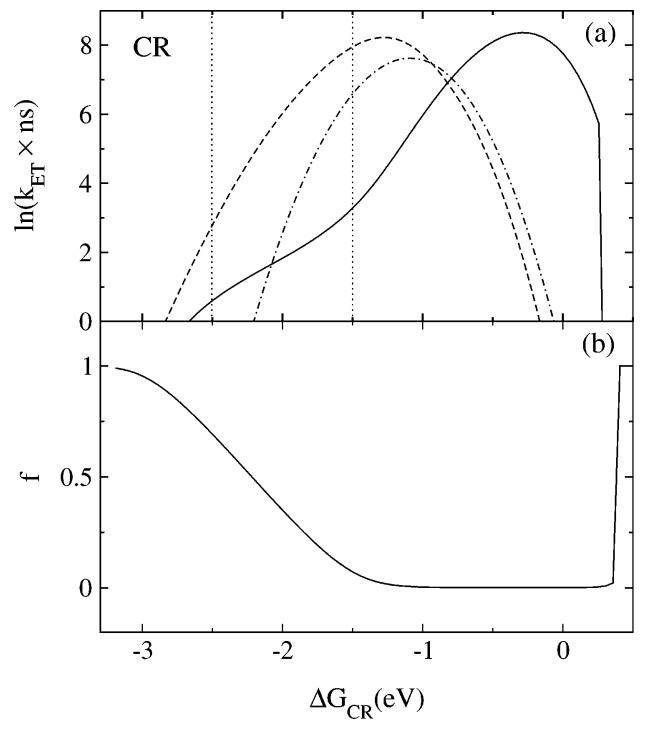

Figure 11. Same as in Figure 10 for charge recombination (CR). The dotted lines in (a) separate the region of the shoulder in the energy gap law, which results in a weak dependence of the rate on the ET matrix element shown in Figure 12.

equation $^{71}$

$$
k_{\mathrm{ET}}^{\mathrm{CS} / \mathrm{CR}}=\left(\frac{2 \pi V_{\mathrm{ET}}^{2}}{\hbar}\right) e^{-\mathrm{S}} \sum_{n} \frac{S^{n}}{n !} G_{n}^{\mathrm{CS} / \mathrm{CR}}
$$

where

$G_{n}^{\mathrm{CS} / \mathrm{CR}}=\left[\frac{4 \pi \lambda_{0}}{\beta}\right]^{-1 / 2} \exp \left[-\beta \frac{\left(\Delta G_{\mathrm{CS} / \mathrm{CR}}+\lambda_{0}+n \hbar \omega_{\mathrm{v}}\right)^{2}}{4 \lambda_{0}}\right]$

and $\lambda_{0}$ is given by eq 29. As established in previous studies of the problem, ${ }^{18}$ the hybrid model used here gives higher rates in the inverted region of ET.

The recombination rate is much more sensitive to nonergodic effects than the charge-separation rate (Figure 11). Several features can be separated here. First, the vertical shift is affected by nuclear solvation, and, as a result, the top of the inverted parabola (solid line) is shifted away from the ergodic calculations (dashed and dash-dotted lines) because $f \simeq 0$ in the region of the parabola's top. Second, the reaction in the normal ET region switches rather sharply between the nonergodic and ergodic regimes, resulting in a very substantial discontinuity of the energy gap law. The position of this turnover point depends on temperature through the Debye relaxation time of the solvent. Third, a shoulder in the energy gap law, corresponding to growing nonergodicity function, develops in the inverted region of ET.

The region of the shoulder in the energy gap law for charge recombination, separated by the dotted lines in Figure 11a, is responsible for an interesting dependence of the rate on the ET matrix element shown in Figure 12. For all other regions of the energy gap law, the logarithmic scale of the rate constant $k_{\mathrm{ET}}^{\mathrm{CR}}$ vs $V_{\mathrm{ET}}$ gives the slope of 2.0, as expected from the Golden Rule expression for the reaction rate (e.g., eq 38). However, in the region of changing nonergodicity function, an increase in the rate due to higher $V_{\mathrm{ET}}$ is partially compensated by a concomitant increase in the activation barrier due to the changing extent of nonergodicity. As a result, the plots shown in Figure 12 have the slopes of 0.81 and 0.65 , instead of 2.0 , for $\Delta G_{\mathrm{CR}}$ equal to $-1.5 \mathrm{eV}$ and $-2.0 \mathrm{eV}$, respectively. When the nonergodicity function (dashed line in Figure 12) becomes close to one, the

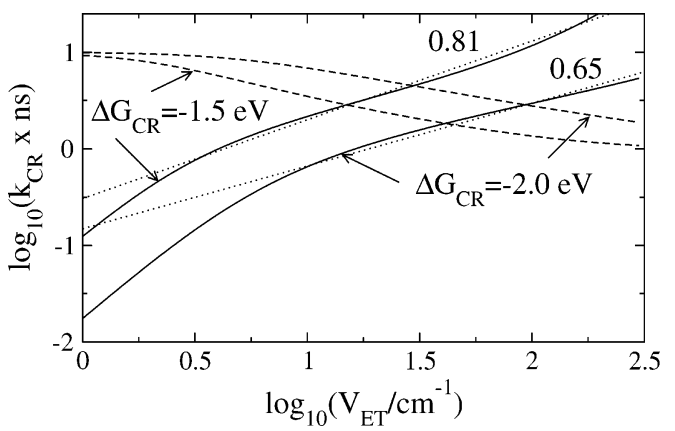

Figure 12. Recombination rates (solid lines) at the free energy gaps indicated in the figure. The dashed lines show the nonergodicity function, and the dotted lines are linear regressions taken for the range of data limited by $\log \left(V_{\mathrm{ET}} / \mathrm{cm}^{-1}\right)>0.75$. The slopes of the linear regressions are indicated in the plot. The solute parameters are the same as in Figure 10.

slope changes to 2.0 , as is seen from the left portion of the plot in Figure 12. If one assumes the commonly observed exponential distance decay of $V_{\mathrm{ET}}^{2}$ with the decay exponent equal to 1 $\AA^{-1},{ }^{76}$ the apparent decay exponents of the rates from Figure 12 will be 0.4 and $0.3 \AA^{-1}$. Therefore, the interpretation of experimental data for the distance dependence of ET in slowly relaxing solvents might require corrections for nonergodicity effects arising from the variation of the observation window caused by the exponential decay of $V_{\mathrm{ET}}$.

\section{Concluding Remarks}

We have studied the energetics of ET reactions in liquid crystalline solvents both in their isotropic and nematic phases. The transition of the solvent to nematic order does not introduce any dramatic changes in the reaction energetics. All changes to the reaction barrier at the point of phase transition are related to anisotropy of solvation energy in the nematic phase in terms of the orientation of the donor-acceptor complex relative to the director. This anisotropy, narrowing with increasing solute size, is mostly a result of microscopic correlations of the solvent dipoles. A much more dramatic effect of the solvent on the energetics is dynamic in nature caused by falling the nuclear solvation out of ergodicity.

The traditional view of the effect of solvent dynamics on activated chemical reactions is based on the ideas advanced in the Kramers model of chemical kinetics. ${ }^{40}$ The modification of the transition state formalism suggested by Kramers anticipates the depletion of the equilibrium population of the reactant states on the top of the activation barrier. The preexponent of the reaction rate is then proportional to the solvent friction or inversely proportional to the relaxation time of the solvent mode driving the transition (solvent-controlled ET, ${ }^{15,16}$ eq 1). The description of faster reactions and/or slow solvents requires further development of these ideas. When the reaction time is comparable to the relaxation time of the solvent, finding the activation barrier requires solving a self-consistent equation for the rate constant (eqs 4 and 32). The activation barrier, instead of the rate preexponent in the Kramers picture, becomes dependent on the solvent dynamics.

Subnanosecond ET reactions in slowly relaxing liquid crystalline solvents present exactly the situation when the solvation component of the activation barrier falls in the nonergodic regime even in the isotropic phase. (There is nothing specific about nematics in this regard, and the same picture applies to any sufficiently sluggish solvent.) All solvation parameters entering the activation barrier gain temperature dependence quite different from what is expected at equilibrium conditions. Both the theoretical calculations and the analysis 
of experimental data indicate that only a small fraction of equilibrium solvation energy enters the activation barrier (Figure 9) for subnanosecond reactions. The fraction of the solvation free energy present in the barrier (nonergodicity function) depends strongly on the rate of ET. This offers a unique opportunity to change the rate by switching the reaction from ergodic to nonergodic behavior. Electrochemistry in liquid crystalline solvents ${ }^{8}$ stands out among possible applications of this phenomenon. The present theory predicts that reaction rates can change quite sharply with changing electrode overpotential in the normal region of ET when the reaction switches between ergodic and nonergodic regimes (Figures 10 and 11). Finally, the interpretation of the ET experiment will require modifications regarding both the activation barrier and the rate distance dependence once nuclear solvation becomes nonergodic.

On a more general note, the present study of various aspects of the solvent effect on ET in liquid crystals (polarization anisotropy, phase transition, slow dynamics) points to a much stronger effect of dynamical nonergodicity on chemical reactivity than of any other solvent parameter, thermodynamic state included. It may turn out that this regulation mechanism is widely exploited by nature in situations where efficiency and precise tuning are at stake. ${ }^{77}$

Acknowledgment. This research was supported by the National Science Foundation (CHE-0304694).

\section{References and Notes}

(1) Marcus, R. A. Rev. Mod. Phys. 1993, 65, 599.

(2) Raineri, F. O.; Friedman, H. L. Adv. Chem. Phys. 1999, 107, 81.

(3) Warshel, A.; Parson, W. W. Q. Rev. Biophys. 2001, 34, 563.

(4) Aviram, A., Ratner, M., Mujica, V., Eds.; Molecular Electronics II; New York Academy of Sciences: New York, 2002; Vol. 960.

(5) Steffen, M. A.; Lao, K.; Boxer, S. G. Science 1994, 264, 810.

(6) Matyushov, D. V. J. Phys. Chem. B 2006, 110, 10095.

(7) Schmid-Mende, L.; Fechtenkötter, A.; Müllen, K.; Moons, E.;

Friend, R. H.; MacKenzie, J. D. Science 2001, 293, 1119.

(8) Rusling, J. F. Acc. Chem. Res. 1998, 31, 363.

(9) Hasharoni, K.; Levanon, H. J. Phys. Chem. 1995, 99, 4875

(10) Wiederrecht, G. P.; Svec, W. A.; Wasielewski, M. R. J. Am. Chem. Soc. 1997, 119, 6199 .

(11) Wiederrecht, G. P.; Svec, W. A.; Wasielewski, M. R. J. Phys. Chem. B 1999, 103, 1386.

(12) Sinks, L. E.; Wasielewski, M. R. J. Phys. Chem. A 2003, 107, 611.

(13) Di Valentin, M.; Bisol, A.; Agostini, G.; Fuhs, M.; Liddell, P. A.; Moore, A. L.; Moore, T. A.; Gust, D.; Carbonera, D. J. Am. Chem. Soc. 2004, 126, 17074.

(14) Sinks, L.; Fuller, M. J.; Liu, W. H.; Ahrens, M. J.; Wasielewski, M. R. Chem. Phys. 2005, 319, 226.

(15) Zusman, L. D. Chem. Phys. 1980, 49, 295.

(16) Weaver, M. J.; McManis, G. E. Acc. Chem. Res. 1990, 23, 294.

(17) Sumi, H.; Marcus, R. A. J. Chem. Phys. 1986, 84, 489.

(18) Walker, G. C.; Aakesson, E.; Johnson, A. E.; Levinger, N. E.; Barbara, P. F. J. Phys. Chem. 1992, 96, 3728.

(19) Bagchi, B.; Gayathri, N. Adv. Chem. Phys. 1999, 107, 1.

(20) Davies, M.; Moutran, R.; Price, A. H.; Beevers, M. S.; Williams,

G. J. Chem. Soc., Faraday Trans. 1976, 72, 1447.

(21) Vertogen, G.; Jeu, V. W. H. D. Thermotropic Liquid Crystals, Fundamentals; Springer-Verlag: Berlin, 1988.

(22) Singh, S. Phys. Rep. 2000, 324, 107.

(23) Sengupta, A.; Fayer, M. D. J. Chem. Phys. 1995, 102, 4193.

(24) Deeg, F. W.; Stankus, J. J.; Greenfield, S. R.; Newell, V. J.; Fayer, M. D. J. Chem. Phys. 1989, 90, 6893.

(25) Urban, S.; Gestblom, B.; Würflinger, A. Mol. Cryst. Liq. Cryst. 1999, 331, 113

(26) Urban, S.; Gestblom, B.; Kuczynski, W.; Pawlus, S.; Würflinger, A. Phys. Chem. Chem. Phys. 2003, 5, 924.

(27) Drozd-Rzoska, A.; Rzoska, S. J. Phys. Rev. E 2002, 65, 041701. (28) Ginovska, M.; Czechowski, G.; Déjardin, J.-L.; Jadzyn, J.; Hellemans, L. Liq. Cryst. 2005, 32, 625.

(29) Dunmur, D., Fukuda, A., Luckhurst, G., Eds.; Physical Properties of Liquid Crystals: Nematics; Exeter: London, 2001.

(30) Lilichenko, M.; Matyushov, D. V. J. Phys. Chem. B 2003, 107, 1937.

(31) Lilichenko, M.; Matyushov, D. V. J. Chem. Phys. 2003, 119, 1559.

(32) Martin, A. J.; Meier, G. Symp. Faraday Soc. 1971, 5, 119.
263.

(33) Urban, S.; Gestblom, B.; Dabrowski, R. Pol. J. Chem. 2002, 76,

(34) Matyushov, D. V. J. Chem. Phys. 2005, 122, 084507.

(35) Hoffman, B. M.; Ratner, M. A. Inorg. Chim. Acta 1996, 243, 233.

(36) Tanimura, Y.; Leite, V. B. P.; Onuchic, J. N. J. Chem. Phys. 2002 $117,2172$.

(37) Kotelnikov, A. I.; Ortega, J. M.; Medvedev, E. S.; Psikha, B. L.; Garcia, D.; Mathis, P. Bioelectrochemistry 2002, 56, 3.

(38) Moran, A. M.; Spears, K. G. Chem. Phys. Lett. 2004, 393, 397.

(39) Leite, V. B. P.; Alonso, L. C. P.; Newton, M. D.; Wang, J. Phys. Rev. Lett. 2005, 95, 118301. 1997.

(41) Ghorai, P. K.; Matyushov, D. V. J. Chem. Phys. 2006, 124, 144510.

(42) Marcus, R. A. J. Phys. Chem. 1990, 94, 4963.

(43) Chen, P.; Meyer, T. J. Inorg. Chem. 1996, 35, 5520.

(44) Gaines, G. L.; O’Neil, M. P.; Svec, W. A.; Niemczyk, M. P.; Wasielewski, M. R. J. Am. Chem. Soc. 1991, 113, 719.

(45) Rhodes, T. A.; Farid, S.; Goodman, J. L.; Gould, I. R.; Young, R. H. J. Am. Chem. Soc. 1999, 121, 5340.

(46) Hansen, J. P.; McDonald, I. R. Theory of Simple Liquids; Academic Press: London, 2003.

(47) Wolynes, P. G. J. Chem. Phys. 1987, 86, 5133.

(48) Fried, L. E.; Mukamel, S. J. Chem. Phys. 1990, 93, 932. 2546

(50) Ghorai, P. K.; Matyushov, D. V. J. Am. Chem. Soc. 2005, 127, 16390 .

(51) Matyushov, D. V. J. Chem. Phys. 2004, 120, 7532.

(52) Kapko, V.; Matyushov, D. V. J. Chem. Phys. 2006, 124, 114904.

(53) Skaf, M. S. J. Chem. Phys. 1997, 107, 7996.

(54) Milischuk, A.; Matyushov, D. V.; Newton, M. D. Chem. Phys. 2006 324,172 .

(55) Ghorai, P. K.; Matyushov, D. V. J. Phys. Chem. A 2006, in press, jp056261i.

(56) Matyushov, D. V. Chem. Phys. 1993, 174, 199

(57) Urano, K.; Inoue, M. J. Chem. Phys. 1977, 66, 791.

(58) Vath, P.; Zimmt, M. B.; Matyushov, D. V.; Voth, G. A. J. Phys Chem. B 1999, 103, 9130.

(59) Saielli, G.; Polimeno, A.; Nordio, P. L.; Bartolini, P.; Ricci, M.; Righini, R. J. Chem. Soc., Faraday Trans. 1998, 94, 121.

(60) Bartolini, P.; Ricci, M.; Righini, R.; Saielli, G.; Polimeno, A.; Nordio, P. L. Mol. Cryst. Liq. Cryst. 1999, 336, 33.

(61) Rau, J.; Ferrante, C.; Kneuper, E.; Deeg, F. W.; Bräuchle, C. J. Phys. Chem. A 2001, 105, 5734.

(62) Urban, S.; Gestblom, B. O.; Dabrowski, R. Phys. Chem. Chem. Phys. 1999, 1, 4843.

(63) Matyushov, D. V. J. Chem. Phys. 2005, 122, 044502

(64) Madden, P.; Kivelson, D. Adv. Chem. Phys. 1984, 56, 467.

(65) Bagchi, B.; Chandra, A. Adv. Chem. Phys. 1991, 80, 1.

(66) Wertheim, M. S. J. Chem. Phys. 1971, 55, 4291.

(67) Frisch, M. J.; Trucks, G. W.; Schlegel, H. B.; Scuseria, G. E.; Robb, M. A.; Cheeseman, J. R.; Montgomery, J. A., Jr.; Vreven, T.; Kudin, K. N.; Burant, J. C.; Millam, J. M.; Iyengar, S. S.; Tomasi, J.; Barone, V.; Mennucci, B.; Cossi, M.; Scalmani, G.; Rega, N.; Petersson, G. A.; Nakatsuji, H.; Hada, M.; Ehara, M.; Toyota, K.; Fukuda, R.; Hasegawa, J.; Ishida, M.; Nakajima, T.; Honda, Y.; Kitao, O.; Nakai, H.; Klene, M.; Li X.; Knox, J. E.; Hratchian, H. P.; Cross, J. B.; Bakken, V.; Adamo, C.; Jaramillo, J.; Gomperts, R.; Stratmann, R. E.; Yazyev, O.; Austin, A. J.; Cammi, R.; Pomelli, C.; Ochterski, J. W.; Ayala, P. Y.; Morokuma, K.; Voth, G. A.; Salvador, P.; Dannenberg, J. J.; Zakrzewski, V. G.; Dapprich, S.; Daniels, A. D.; Strain, M. C.; Farkas, O.; Malick, D. K.; Rabuck, A. D.; Raghavachari, K.; Foresman, J. B.; Ortiz, J. V.; Cui, Q.; Baboul, A. G.; Clifford, S.; Cioslowski, J.; Stefanov, B. B.; Liu, G.; Liashenko, A.; Piskorz, P.; Komaromi, I.; Martin, R. L.; Fox, D. J.; Keith, T.; Al-Laham, M. A.; Peng, C. Y.; Nanayakkara, A.; Challacombe, M.; Gill, P. M. W.; Johnson, B.; Chen, W.; Wong, M. W.; Gonzalez, C.; Pople, J. A. Gaussian 03; Gaussian, Inc.: Pittsburgh, PA, 2003.

(68) Jorgensen, W. L.; Maxwell, D. S.; Tirado-Rives, J. J. Am. Chem. Soc. 1996, 118, 11225 .

(69) Matyushov, D. V.; Schmid, R. J. Chem. Phys. 1995, 103, 2034

(70) Jortner, J.; Bixon, M. J. Chem. Phys. 1988, 88, 167.

(71) Bixon, M.; Jortner, J. Adv. Chem. Phys. 1999, 106, 35.

(72) Nadler, W.; Marcus, R. A. J. Chem. Phys. 1987, 86, 3906.

(73) Greenfield, S. R.; Svec, W. A.; Gosztola, D.; Wasielewski, M. R. J. Am. Chem. Soc. 1996, 118, 6767.

(74) Hasharoni, K.; Levanon, H.; Greenfield, S. R.; David, J. G.; Svec, W. A.; Wasielewski, M. R. J. Am. Chem. Soc. 1996, 118, 10228.

(75) Galili, T.; Regev, A.; Levanon, H.; Schuster, D. I.; Guldi, D. M. J. Phys. Chem. A 2004, 118, 10632.

(76) Newton, M. D. Adv. Chem. Phys. 1999, 106, 303 551 